\title{
Physics at the Thomas Jefferson National Accelerator Facility
}

\author{
L.S. Cardman ${ }^{\mathrm{a}}$
}

Thomas Jefferson National Accelerator Facility, 12000 Jefferson Avenue, Newport News, VA 23606, USA and University of Virginia, Department of Physics, 382 McCormick Rd., P.O. Box 400714, Charlottesville, VA 22904-4714, USA

/ Published online: 31 May 2006 - C) Società Italiana di Fisica / Springer-Verlag 2006

\begin{abstract}
The Continuous Electron Accelerator Facility, CEBAF, located at the Thomas Jefferson National Accelerator Facility, is devoted to the investigation of the electromagnetic structure of mesons, nucleons, and nuclei using high energy, high duty-cycle electron and photon beams. Selected experimental results of particular interest to the MAMI community are presented.
\end{abstract}

PACS. 29.17. $+\mathrm{w}$ Electrostatic, collective, and linear accelerators - 25.20.-x Photonuclear reactions 25.30.Bf Elastic electron scattering - 25.30.Dh Inelastic electron scattering to specific states

\section{Personal Comments}

It is an honor and a pleasure to be here to celebrate the achievements of MAMI and the distinguished careers of Professors Arenhövel, Backe, Drechsel, Friedrich, Kaiser, and Walcher.

We are all deeply aware of the extent to which the science we do builds on the achievements of those who have gone before us, and on the insights and hard work of our colleagues working in the field today. One of my very earliest memories as a scientist, dating from the days when I was a young graduate student, is that of attending "Photonuclear Physics Boot Camp" (otherwise known as the Photonuclear Gordon Conference) and learning from (and with) many of those "retiring" today.

Thomas (Walcher) was one of the very first scientists I ever knew beyond the boundaries of my own laboratory. He came to visit us (at Yale), and I went and visited him and his colleagues at Darmstadt. It has been a great pleasure to follow his distinguished career in science, from low$Q^{2}$ electron scattering to hadronic physics at CERN and beyond, and finally to the leadership role he has played at MAMI for many years.

Hartmuth (Arenhövel) has been the keeper of the flame of all knowledge about the deuteron, and a worthy successor to Gregory Breit. You should know that I was a graduate student at Yale, and it was one of Professor Breit's missions in life to convince any and all who would listen that the deuteron was the essence of nuclear physics, and that until we understood the deuteron, we did not understand anything. I think it is fair to call Hartmuth the Gregory Breit of my generation; he has made so many contributions.

\footnotetext{
a e-mail: cardman@jlab.org
}

Dieter (Drechsel) has always been one of those people I have looked to as the source of the "big picture" in nuclear physics. He has provided us with deep insights, a sense of direction, and an understanding of what is really important. He has also been an inspiring example here at Mainz of the tremendous benefits to everyone of having a close collaboration between theory and experiment.

Jörg (Friedrich) has taught us all how to analyze and interpret electron scattering data with minimal prejudice (and, therefore, maximal honesty). It is a delight to see the same rigorous approach that was so successful in the study of nuclei and their excited states now being applied to nucleon structure.

Karl-Heinz (Kaiser) and his mentor, Helmut Herminghaus, taught the world how to build superb continuouswave (cw) electron accelerators effectively and efficiently. Karl-Heinz, in particular, through the design and construction of the double-sided microtron, is leaving the Institute well positioned for another generation of superb experiments.

In conclusion, on behalf of so many people I have worked with in nuclear physics, I want to thank each of you for your many contributions to our field, and to express the hope we all share that for each of you "retirement" is a formality, not a reality, and that you will continue to be active for years to come.

\section{Research at Jefferson Laboratory}

The Thomas Jefferson National Accelerator Facility, also called Jefferson Lab (or JLab), operates the Continuous Electron Beam Accelerator Facility (CEBAF). CE$\mathrm{BAF}$ is a $\mathrm{cw}$ electron accelerator capable of delivering three electron beams for simultaneous experiments in the 
three experimental areas. Originally designed for $4 \mathrm{GeV}$, its present maximum energy is $5.7 \mathrm{GeV}$.

The CEBAF user community consists of about 2000 physicists; more than half of them are actively involved in the experimental program. In addition to its main mission, JLab contributes to the development and use of Free Electron Lasers, to medical imaging, and to community outreach programs.

The intellectual and technical foundations for the construction of CEBAF were provided by the scientific successes of earlier electron accelerators (the generation that included Saclay, MIT-Bates, NIKHEF, and, to some extent, SLAC), and by the enhanced research opportunities provided by cw electron beams as demonstrated at facilities such as MAMI. CEBAF is a large, international laboratory with a broad research program; it has been in operation for some seven and a half year now.

What are the goals of CEBAF's research program? Basically, we aim to understand strongly-interacting matter. How are the hadrons constructed from the quarks and gluons of QCD, and how does the nucleon-nucleon force arise from the strong interaction? We further aim to identify the limits of our understanding of nuclear structure by using the high precision attainable with the electromagnetic probe and the possibiltiy of extending investigations to very small distance scales. A specific issue that motivated the construction of CEBAF was our desire to gain insight into the question of where the description of nuclei based on nucleon and meson degrees-of-freedom fails and the underlying quark degrees-of-freedom must be taken into account. One can ultimately characterize all of this as trying to understand QCD, not in the perturbative regime accessible at very high energies and very short distance scales, but in the strong interaction regime relevant to most of the visible matter in the Universe. To make progress in these areas, there are other critical issues that must be addressed, such as the mechanism of confinement, the dynamics of the quark interaction, and how chiral symmetry breaking occurs.

To provides some shape and structure to the discussion of the experiments, the CEBAF program can be organized into half a dozen broad thrusts. This presentation will concentrate on two of them:

- How are the nucleons made from quarks and glue?

- Where are the limits of our understanding of nuclear structure

\section{How are the nucleons made from quarks and glue?}

Among the most interesting puzzles in physics today are: why there is this effective degree-of-freedom in QCD, the nucleon; and how something as complicated as the residual QCD interaction between quarks in nucleons can be characterized by a rather simple N-N potential? To provide experimental insights that will help us solve the first of these puzzles, the Jefferson Lab research community has mounted an array of investigations in three broad areas:
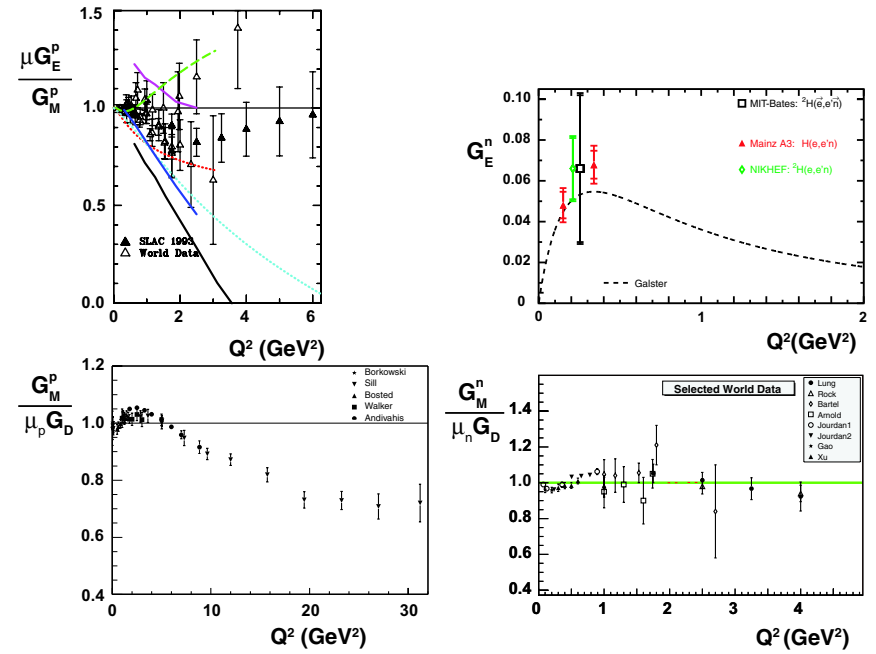

Fig. 1. Nucleon form factor data available before the start of experiments using CEBAF. Top 2 panels: electric form factors, bottom panels: magnetic form factors. Left 2 panels: proton form factors, right panels: neutron form factors (adapted from ref. [1]).

- What are the spatial distributions of the $u, d$, and $s$ quarks in the hadrons?

- What is the excited state spectrum of the hadrons, and what does it reveal about the underlying degreesof-freedom?

- What is the QCD basis for the spin structure of the hadrons?

\subsection{What are the spatial distributions of the $u, d$, and $s$ quarks in the hadrons?}

Elastic electron scattering has provided most of our information on the spatial distributions of the quarks in the nucleons. The data on the four electromagnetic structure functions of the nucleon, $G_{E}$ and $G_{M}$ for both the proton and the neutron, available just prior to the start of experiments at CEBAF is shown in fig. 1. The magnetic form factors of the proton and the neutron were known reasonably well, but the electric form factors were not. The electric form factor of the proton had not been determined accurately enough to distinguish between a wide range of theories based on rather different physics. First results on the electric form factor of the neutron were available from Bates, Mainz, and NIKHEF, but these data were limited to moderate momentum transfers and, therefore, not sensitive to the details of the distribution of charge inside the neutron. The measured form factor was consistent with the r.m.s. radius derived from neutron-electron scattering.

The present status of the nucleon form factors is shown in fig. 2. The measurements of the polarization transfer from the incident electron to the elastically recoiling proton have shown that the electric and magnetic form factors for the proton differ substantially. The systematic differences between the polarization transfer data and the Rosenbluth results for $G_{E} / G_{M}$ are likely 

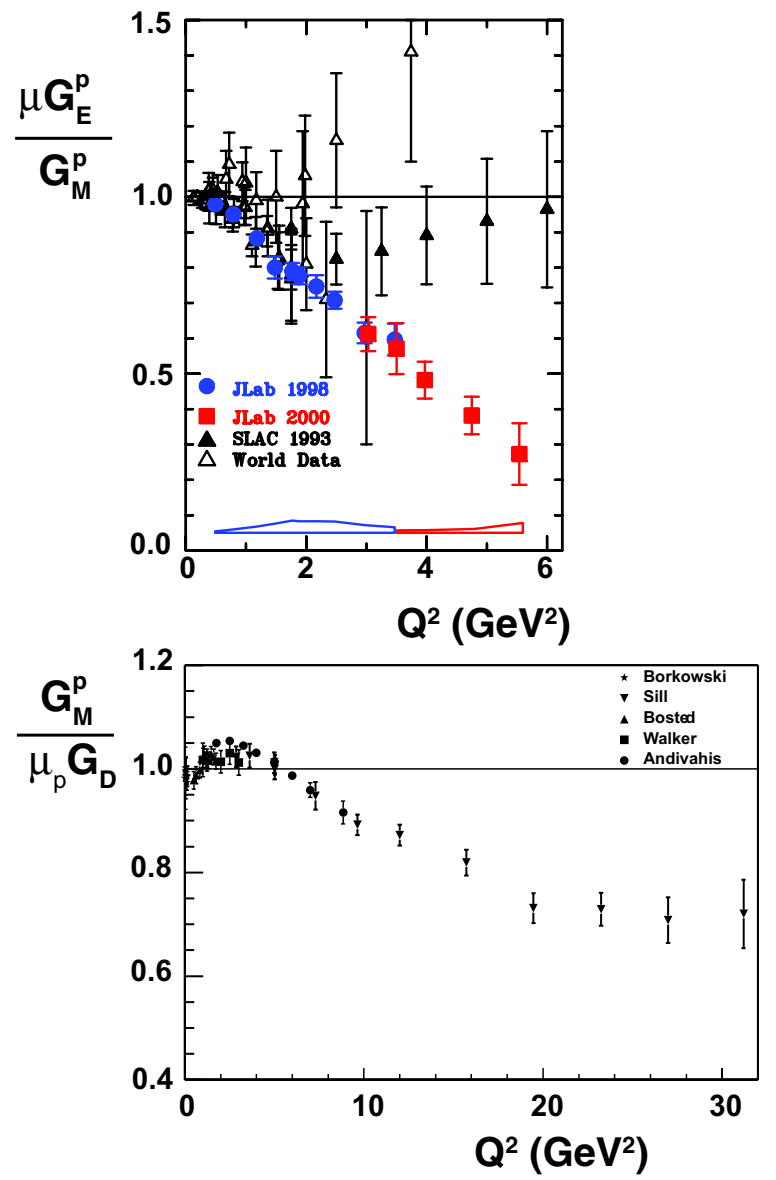
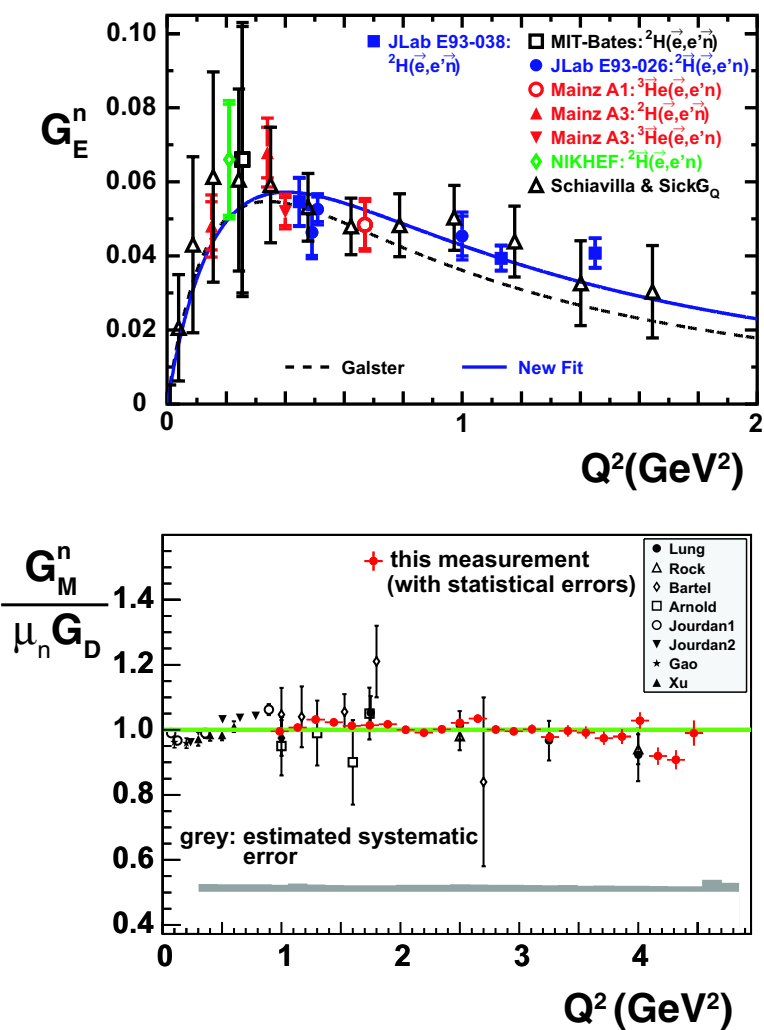

Fig. 2. Present status of the nucleon form factor data including the CEBAF data (adapted from ref. [1]).

due to two-photon exchange effects modifying the results. Theoretical estimates suggest that the modifications are much smaller for the polarization transfer data than for the Rosenbluth data, so the former are likely to be more directly interpretable in terms of the nucleon form factors. The electric form factor of the neutron has now been measured up to a $Q^{2}$ of $1.5(\mathrm{GeV} / c)^{2}$ using polarization transfer techniques, and the data taken with different methods agree quite well.

The theoretical interpretation of the data is summarized in fig. 3. The theories that describe the data reasonably well reveal two key aspects of nucleon structure: the importance of the pion cloud, and the importance of incorporating the relativistic motion of the quarks into the theoretical description of the nucleon.

When one looks at these form factors in a phenomenological way with minimum prejudice [2], what emerges is some of the clearest evidence we have for the nucleon's pion cloud (see fig. 4). Similar results have been obtained using a different approach to model-independent analysis [3] of nucleon form factors.

We plan to extend the proton form factor data to $\sim 9(\mathrm{GeV} / c)^{2}$, where we may see evidence for a diffraction minimum. The neutron form factor will also be extended to $\sim 5(\mathrm{GeV} / c)^{2}$. Further extensions of a factor of two are planned with the $12 \mathrm{GeV}$ Upgrade. Such extensions have
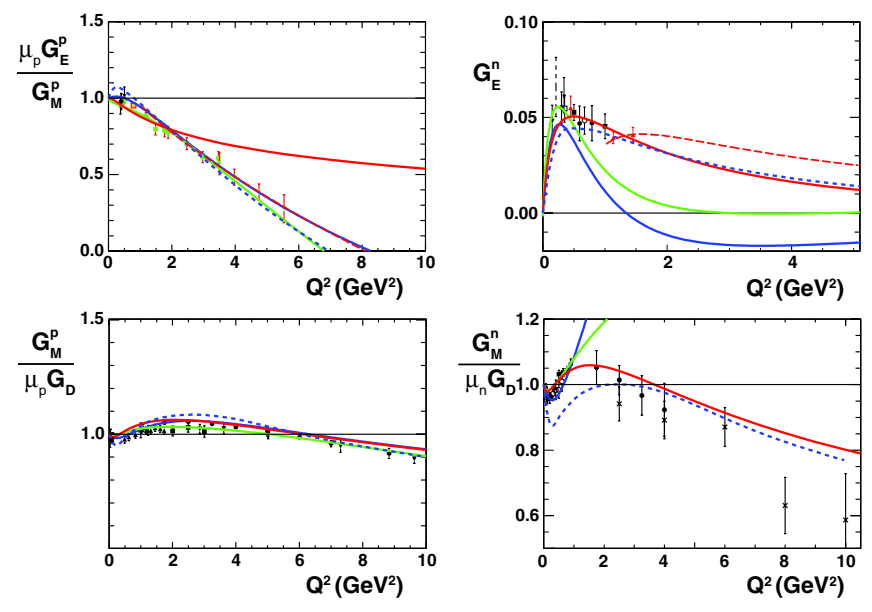

- Bijker (VMD)

- Holzwarth (soliton)

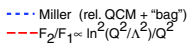

Fig. 3. Theoretical descriptions of the nucleon form factor data [1].

historically proven to be important, and we expect these data will provide further insight and sensitivity for completing our understanding of how to construct nucleons from quarks and gluons. 

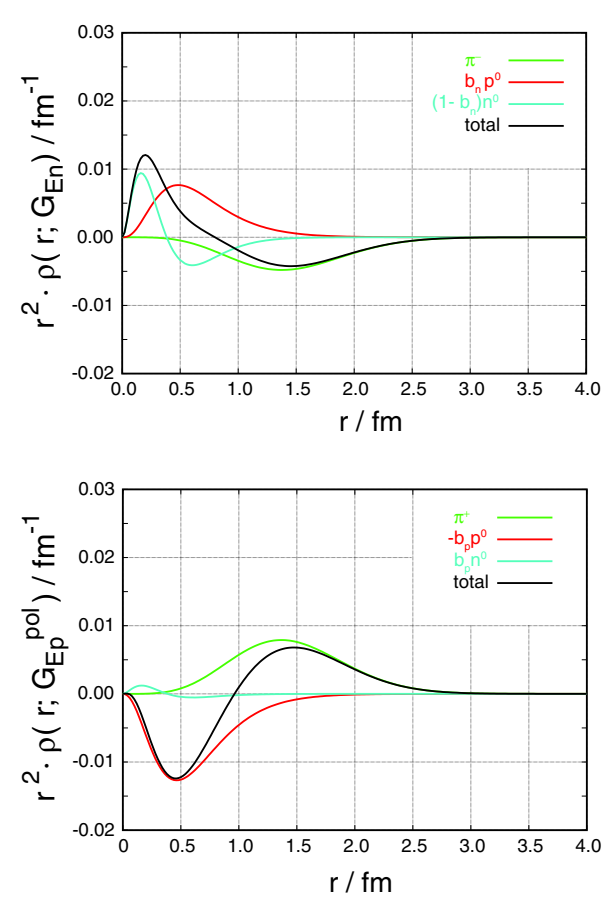

Fig. 4. Neutron radial charge distribution (top) and the polarization term contribution to the proton radial charge distribution (bottom) as inferred from an analysis using phenomenological models [2] of the world nucleon form factor data. The green solid lines show the pion cloud contribution. Note that $r^{2} \rho(r)$ is plotted, emphasizing the distribution at large radii.

The strange quark form factors have become an interesting area of study that is both analogous and complementary to the classical electromagnetic form factors. By using the weak component of the electro-weak interaction we access the weak neutral current form factor, which can be interpreted very elegantly in terms of the strange quark distribution. Because there are no valence strange quarks, this measurement provides a unique window on the sea quark distribution. The strange form factors can also be expected to provide us with interesting experimental insights into nucleon structure: by combining the electromagnetic and the weak neutral current form factors we should be able to separate the spatial distribution of the $u, d$, and $s$ quarks.

Figure 5 shows the world's data on the strange proton form factor taken at forward angles as a function of $Q^{2}$. One sees data from the A4 experiment at Mainz $[6,7,8]$, from HAPPEx I and II (JLab Hall A) [9, 10,11], and from G0 (JLab Hall C) [13]. These difficult experiments would be impossible without highly polarized electron beams from magnificently stable accelerators. The fact that the data from different laboratories lie roughly on a smooth curve gives one confidence that the experimenters are doing it right.

The first thing that strikes you about the data is that the form factor is rather small. This is to be expected, as all of the strange quarks emerge as quark-antiquark pairs popping in and out of the vacuum, and to get a finite form factor there must be some kind of a polarizing effect

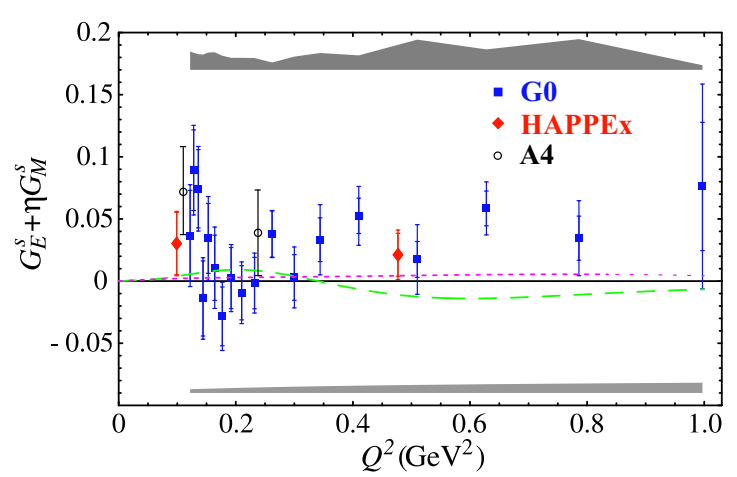

Fig. 5. Nucleon strange form factor data from parity-violating electron scattering at forward angles as a function of $Q^{2}$.

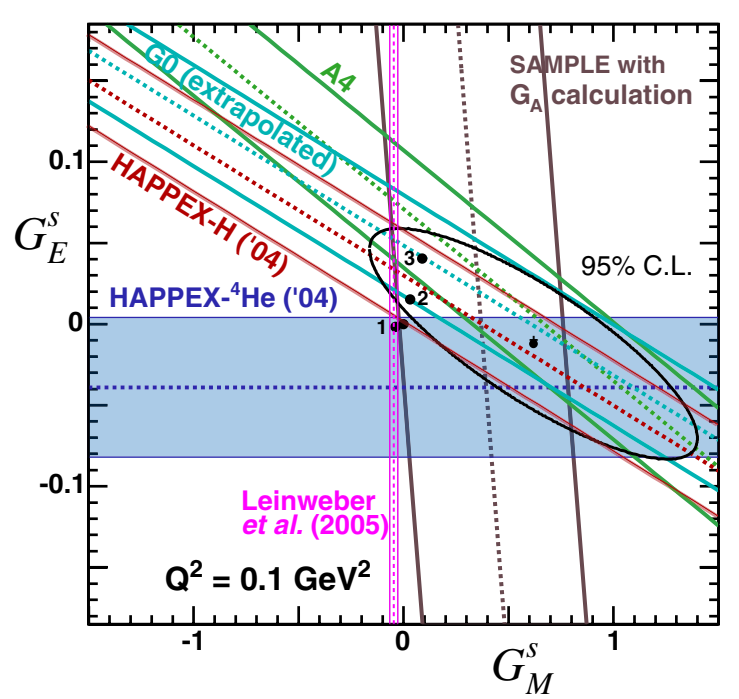

Fig. 6. Separated strange nucleon form factors at $Q^{2}=$ $0.1(\mathrm{GeV} / c)^{2}[10]$.

separating them spatially. Even at the highest momentum transfers reached experimentally we are averaging over a distance scale that is roughly the size of the nucleon, so it is not too surprising that the result is small. There is an intriguing suggestion in the data for something that one would call vaguely pion-cloud like behavior, but it is fair to say that the statistical significance of this effect is not very high.

The data taken at forward angles includes a mixture of electric and magnetic form factors. At $Q^{2}=0.1(\mathrm{GeV} / c)^{2}$ we have data at both forward and backward scattering angles, so we can separate these effects (see fig. 6). The data favor a positive value of $G_{M}^{s}$, which is at variance with most of the theoretical models. Experiments are in progress that will reduce the size of the error ellipse at this $Q^{2}$ value by a about a factor of 3 , and additional experiments planned at both MAMI and JLab will permit separations at other $Q^{2}$ values. A broad, world-wide effort will provide the results we want.

Another interesting experiment is the measurement of the pion form factor. The pion is the simplest QCD bound system, the "positronium" of QCD. One expects that the pion form factor will provide us with evidence for the 


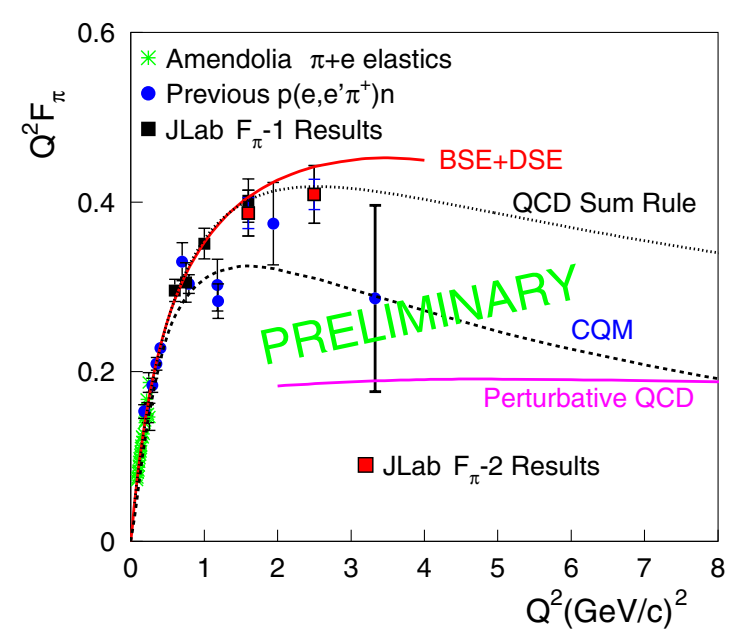

Fig. 7. Pion form factor data [14].

transition of the strong interaction from the perturbative (QED-like) to the strong (confinement) regime at the lowest possible momentum transfer. These data also constrain phenomenological models of the pion.

Measuring the pion form factor is not simple. At low $Q^{2}$, one can scatter pions off atomic electrons, but a bowling ball does not transfer energy to a ping-pong ball efficiently, and even with very high energy pions this experiment cannot reach high momentum transfers. To reach higher momentum transfer in the absence of a free pion target, one must scatter electrons off virtual pions inside a proton and extrapolate the data to the pion pole. The world's data (see fig. 7) is beginning to distinguish between different theoretical approaches. With the $12 \mathrm{GeV}$ Upgrade, we expect to extend the data out to a momentum transfer of $6(\mathrm{GeV} / c)^{2}$ and to be able to infer the distance scale for the onset of perturbative behavior.

\subsection{What is the excited state spectrum of the hadrons?}

If one looks at several decades worth of data on nucleon resonances and tries to use a simple quark model to classify the states in terms of the excitation in units of $\hbar \omega$ and the angular momentum of the three quarks, the states that have been identified so far fit nicely into this scheme, but there are many states that have been predicted but have not been found. It is an interesting fact that one can explain all of the states that have been seen so far by assuming that the nucleon and its excited states are a diquark-quark system. Since most of the data have been obtained from pion-induced reactions, and many of the missing states are predicted not to couple to pions, it is also possible that the missing states may have been overlooked for experimental reasons.

In atomic spectroscopy the line spacing is large compared to the line width, and measuring the complete spectrum is relatively straightforward. In nucleon spectroscopy, the strong interaction causes the width to be comparable to the spacing. Identifying weak states and

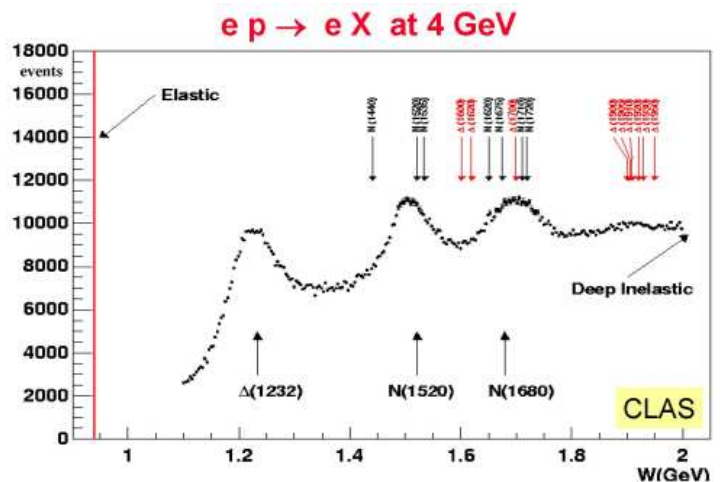

Fig. 8. W-dependence of the scattered electron rate for the $p\left(e, e^{\prime}\right) X$ reaction. CLAS data taken at $4 \mathrm{GeV}$ primary beam energy. The energies of the known excited states are shown in black, while those of the states "missing" in the simple quark model description are shown in red.

extracting the internal quark structure from the measured cross sections is a difficult task. The problem can be seen easily in fig. 8, which shows the inclusive electron scattering cross spectrum from the proton for a $4 \mathrm{GeV}$ electron beam. With a modern electron accelerator and a large acceptance detector one can obtain data on the transition form factors over a large $Q^{2}$-range $\left[1 \rightarrow 4(\mathrm{GeV} / \mathrm{c})^{2}\right]$ in a single shot. There is plenty of cross section in the region where the missing states (shown in red) have been predicted, but extracting their individual strengths from the data is a real challenge.

The combination of cw electron beams and modern, large solid angle detectors provides important advantages for addressing this problem experimentally. If one looks at the same data set of fig. 8 but uses the information on the energy and momentum of the final state proton measured in coincidence with the inelastically scattered electron, it is straightforward to infer the missing mass associated with the decay of the excited state (see fig. 9). One can see clearly from the raw missing mass spectrum that the "missing" states do not couple to pions, but rather to the $\eta$ and $\omega$. With the further information on the angular correlations of those decay particles relative to the momentum transfer axis one finally has the information necessary to decompose the spectrum of fig. 8, and learn just what is there.

This effort naturally begins with the $\Delta(1232)$, which decays predominantly into pion and nucleon. Figure 10 shows a comparison of separated structure functions from CLAS data for the $p\left(e, e^{\prime} p\right) \pi^{o}$ reaction with theoretical fits and results from previous experiments.

The $\Delta \rightarrow \gamma^{*} \mathrm{~N}$-transition is characterized by three multipoles: the electric quadrupole $E$, the magnetic dipole $M$, and the scalar multipole $S$. As we examine this transition as a function of momentum transfer we expect that different aspects of the excitation will become apparent. At large distance scales (corresponding to low momentum transfers) we should see the effect of the pion cloud, while at large momentum transfer (corresponding to short distances) we will eventually reach the limit given by pQCD 


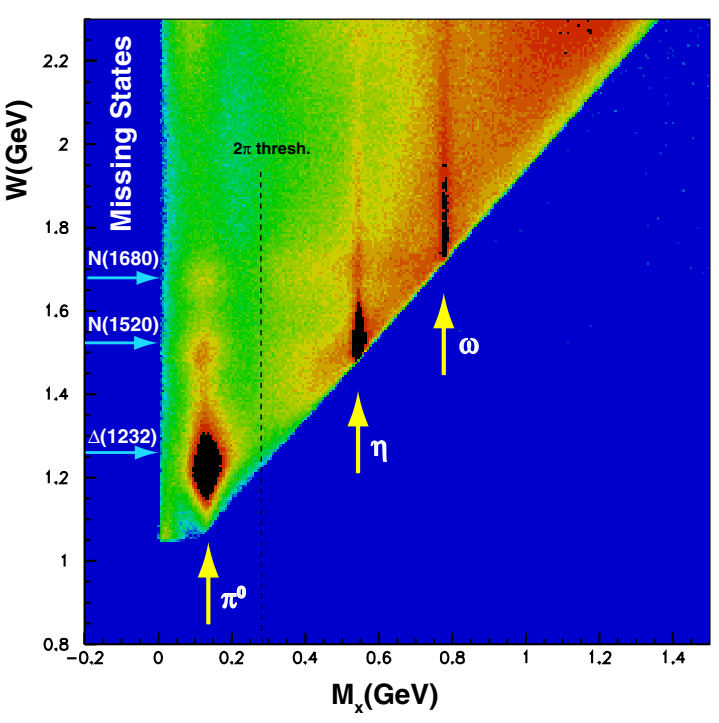

Fig. 9. $W$ vs. missing-mass $M_{x}$ for the same CLAS data shown in fig. $8[15]$.

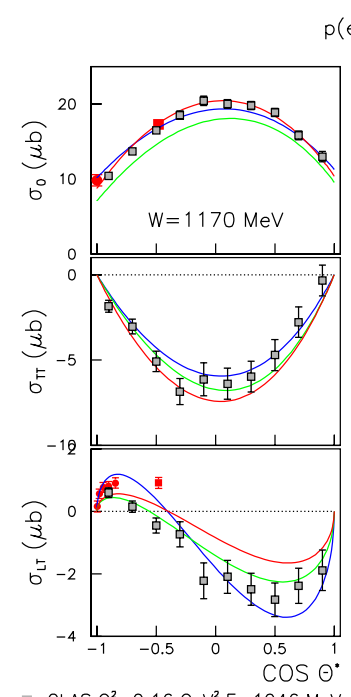

$p\left(e, e^{\prime} p\right) \pi^{0}$

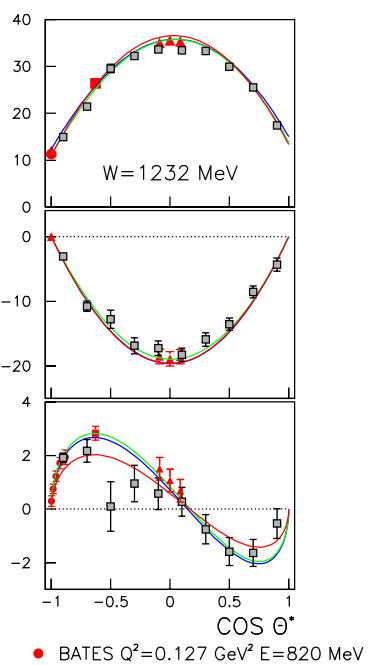

Fig. 10. Angular distributions of the separated structure functions for the $p\left(e, e^{\prime} p\right) \pi^{o}$ reaction in the Delta region [16].

where $R_{E M}=E / M \rightarrow 1$, and we further expect that the $S / M$ ratio $R_{S M}$ will become constant.

Results from an early experiment at JLab and data from MAMI and Bates, all in the low- $Q^{2}$ regime show the effect of the pion cloud clearly (see fig. 11). As a function of $Q^{2}, R_{E M}$ remains small and negative at high $Q^{2}$ with a trend toward 0 and a possible sign change. $R_{S M}$ continues to rise in magnitude with $Q^{2}$. No trend is seen towards $Q^{2}$ independence. We can only conclude that even at $Q^{2}$ of $10(\mathrm{GeV} / c)^{2}$ we are far from the pQCD regime. Pion cloud models describe the data well (fitted to low and high- $Q^{2}$ points). Unquenched Lattice QCD gives the correct signs and approximate magnitudes.

One of the most interesting examples of the impact of the pion cloud and of the value of measuring the transition form factors for nucleon excitation is the Roper

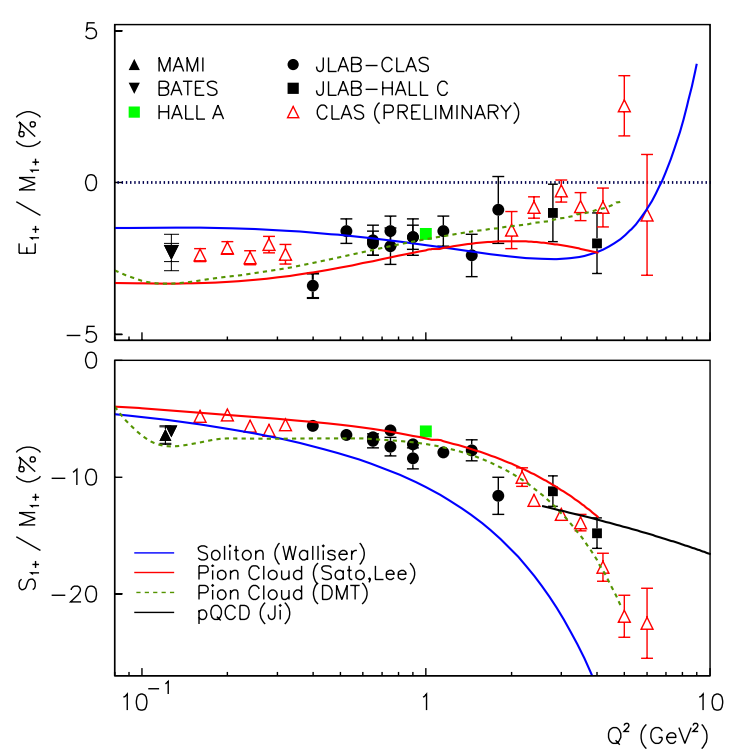

Fig. 11. Ratios $R_{E M}$ and $R_{S M}$ as a function of $Q^{2}$ for the $\Delta \rightarrow \gamma * N$-transition [17].
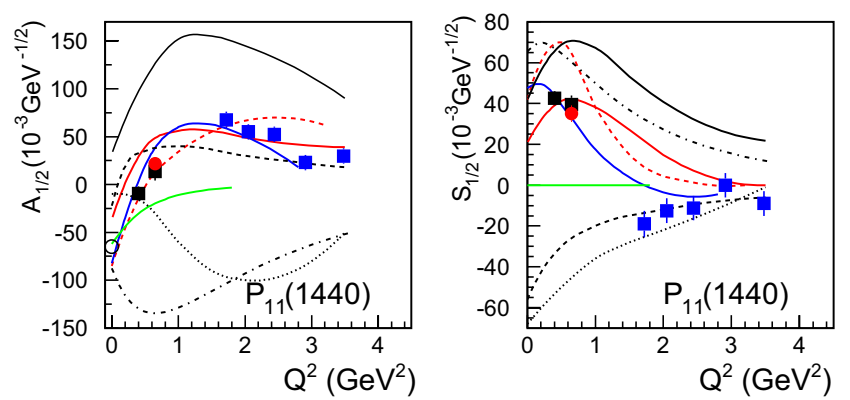

Fig. 12. $A_{\frac{1}{2}}\left(Q^{2}\right)$ and $S_{\frac{1}{2}}\left(Q^{2}\right)$ multipoles for the $P_{11}(1440)$ Roper resonance [18].

resonance. According to the constituent quark model the $\mathrm{N}^{*}(1440) P_{11}$ state is an $N=2$ radial excitation of the nucleon. However, the properties of this state such as its mass and photocouplings are not well described by this model. The new CLAS data (see fig. 12) seem to explain this puzzle. At low momentum transfer, what one is measuring is dominated by the pion cloud. As you start squeezing down the distance scale, what emerges is the underlying quark structure of the Roper, which is, in fact, roughly consistent with a radial excitation.

Investigation of nucleon excitation through the measurement of the transition form factors is now slowly moving up in excitation energy. Most of this analysis is at a preliminary stage, and what is really needed is a coherent study of many channels at many values of momentum transfer in a consistent (and comprehensive) analysis. It will be a long time before we have all the answers.

As we search through this data, we are coming across intriguing evidence for states that have been "missing". For example, there is evidence for a possible new $\mathrm{N}^{*}$ state near $1840 \mathrm{MeV}$ visible in the $\Lambda$ photo- and electroproduction data. In the forward hemisphere, one sees a nice peak from a known $\mathrm{N}^{*}$ state at $1.7 \mathrm{GeV}$; in the 


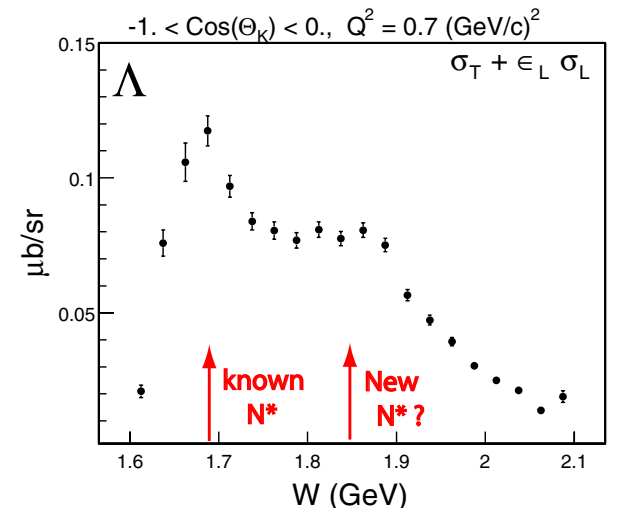

Fig. 13. W-dependence of the cross section for the $p\left(e, e^{\prime} K^{+}\right) \Lambda$ reaction integrated over backward-going $K^{+}[15]$.

backward hemisphere (see fig. 13), one sees an additional unexpected structure. A detailed analysis shows that the angular distribution can be fit nicely with the addition of a new $P_{11}$ state at $1840 \mathrm{MeV}$ with a width $\Gamma=140 \mathrm{MeV}$ to the known $\mathrm{D}_{13}(1870)$ and $\mathrm{D}_{13}(2170)$ states. Intriguingly, a $P_{11}$ state at $1840 \mathrm{MeV}$ is consistent with the symmetric quark model and $S U(6) \times O(3)$ symmetry, but is inconsistent with diquark-quark symmetry.

I feel obliged to bring you up to date on the pentaquark (or lack thereof). There was a great deal of excitement for a while about what appeared to be evidence for a 5-quark state. There have three experiments at JLab pushing to substantially higher statistics, both in the $\gamma p$ and the $\gamma d$ channel, and for virtual photons as well. No evidence for a 5-quark state has been found in the first analyses of these new data.

\subsection{What is the QCD basis for the spin structure of the hadrons?}

In addition to the investigation of the spatial distributions of charge and magnetization in the nucleon and its excited state spectrum, the third important experimental focus is the nucleon's spin structure. The first thing to look at is the spin structure function of the valence quarks at high$x$. The data for the proton was reasonable; the new CLAS data with somewhat tighter error bars are confirming the old results and improving the overall accuracy. There were no data of any statistical significance for the neutron above an $x$ of 0.3 . The ${ }^{3} \mathrm{He}$ experiment at JLab has provided three new data points (see fig. 14). The new data, when folded into a global analysis of the parton distribution functions (PDF), show that the theoretical prejudices used in earlier analyses were wrong; in particular we now know that $\Delta d / d$ stays negative at high $x$.

One can make predictions with a minimum of theoretical prejudice for the integrals of the spin structure functions at the two extremes of distance scales. In the limit of extremely small distances (i.e. for $Q^{2} \rightarrow \infty$ ), assuming only isospin symmetry and current algebra (or the operator product expansion within QCD), Bjorken showed that
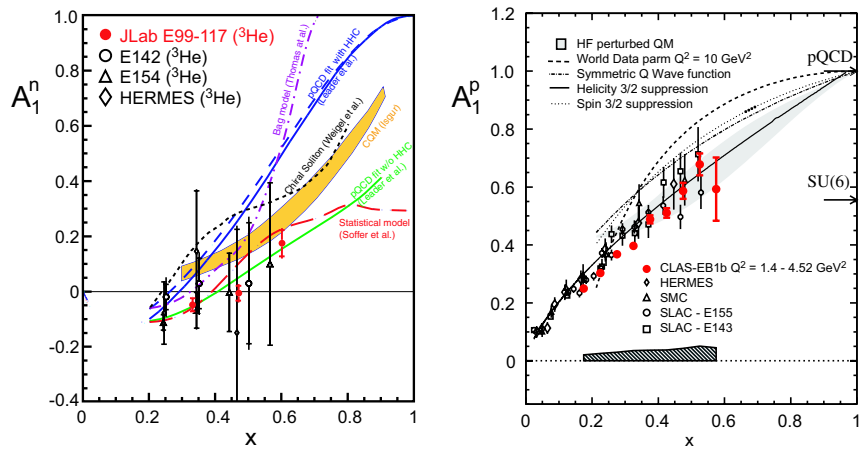

Fig. 14. (Left) Spin structure function of the neutron, $A_{1}^{n}$, derived from ${ }^{3}$ He data [19]; and (Right) spin structure function of the proton $A_{1}^{p}[20]$.

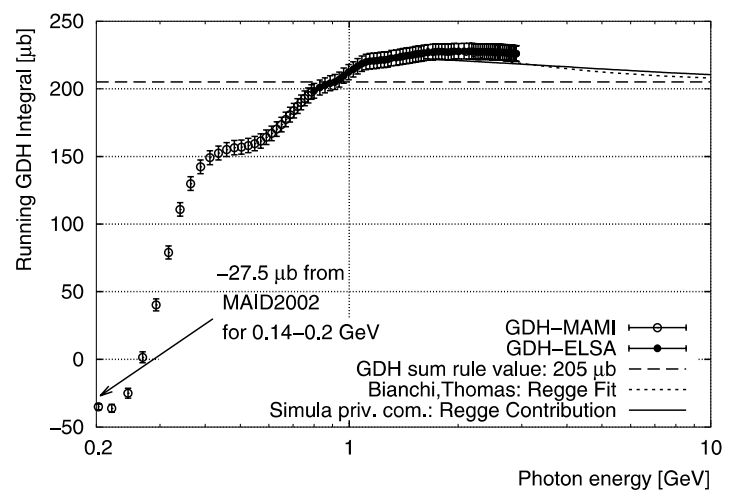

Fig. 15. GDH integral as a function of the upper limit $\nu_{\max }[21]$.

the difference between the proton and the neutron integrals is related to the neutron $\beta$-decay coupling constant, with a small $Q^{2}$ dependent correction due to the running of the coupling constant.

For very large distance scales $\left(i . e\right.$. for $Q^{2} \rightarrow 0$ ), there is a slightly less rigorous set of assumptions (Lorentz invariance, gauge invariance, unitarity, and the dispersion relation applied to the forward Compton amplitude) that can be used to show that the difference between the helicity $\frac{3}{2}$ and $\frac{1}{2}$ total cross sections is related to the nucleon anomalous magnetic moment (this is the GDH sum rule).

There has been a lovely set of data taken at ELSA and MAMI that have determined the GDH integral as a function of the upper photon energy integration limit (see fig. 15). The experiments were technically challenging [21], requiring the combination of polarized electrons, a polarized target, and large-acceptance detectors. Theoretical analysis and interpretation of these data show that the GDH sum rule is satisfied at the $5 \%$ level. The effort has also provided us with a better understanding of the physics of the reactions contributing to the integral. These data, and the precision with which they have defined the GDH integral at the photon point, provide the foundation for our studies of the $Q^{2}$ evolution of the moment of the nucleon's spin structure functions.

As one looks at the evolution of the moment of the proton spin structure function with $Q^{2}$, one expects to see 


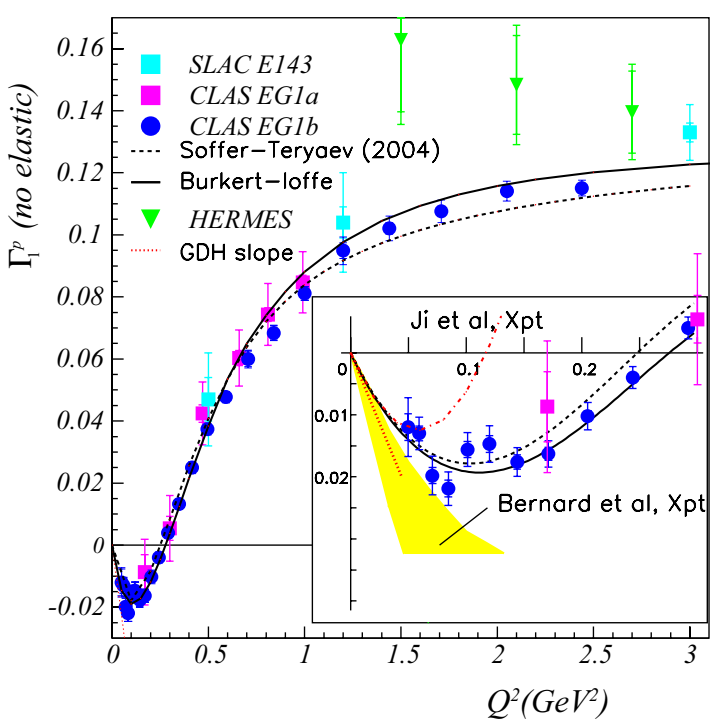

Fig. 16. Integral of the spin structure function of the proton as a function of $Q^{2}[22]$.

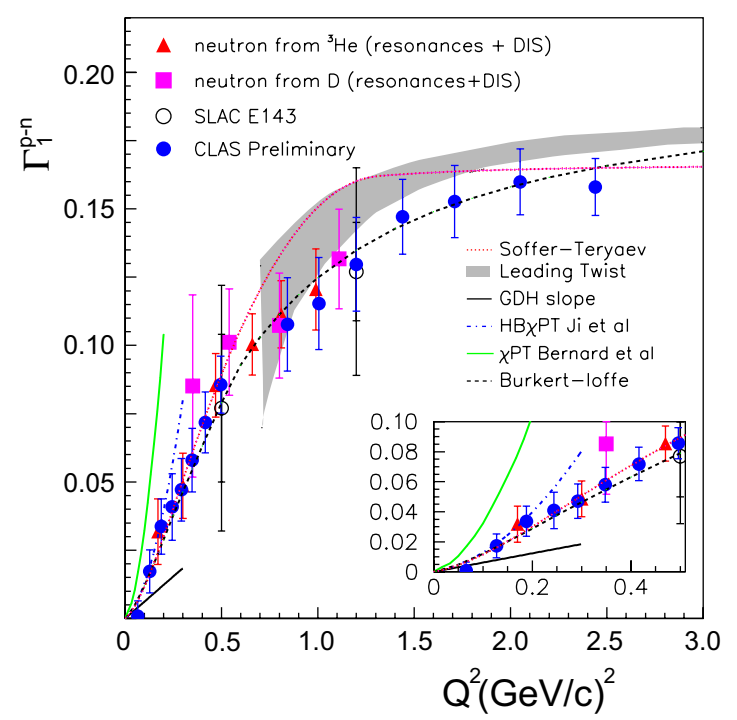

Fig. 17. Bjorken integral (proton-neutron difference) as a function of $Q^{2}$. The grey band shows the evolution of $\alpha_{e f f}\left(Q^{2}\right)$ predicted by pQCD [22].

the anomalous magnetic moment of the proton in the long wavelength limit, whereas at infinite $Q^{2}$ the Bjorken sum rule is valid. In the regime close to the long wavelength limit, chiral perturbation theory $(\chi \mathrm{PT})$ allows us to make predictions. The transition between the two extremes is an important piece of information on how the nucleon is put together, and how nucleon structure emerges from the parton soup.

We have data now, mainly from JLab, on the evolution of the structure function's integral for the proton (see fig. 16) and the neutron approaching the GDH sum rule limit at $Q^{2}=0$, and approaching the Bjorken limit at a surprisingly low momentum transfer of about $1(\mathrm{GeV} / c)^{2}$. Several experiments at JLab are investigating the region

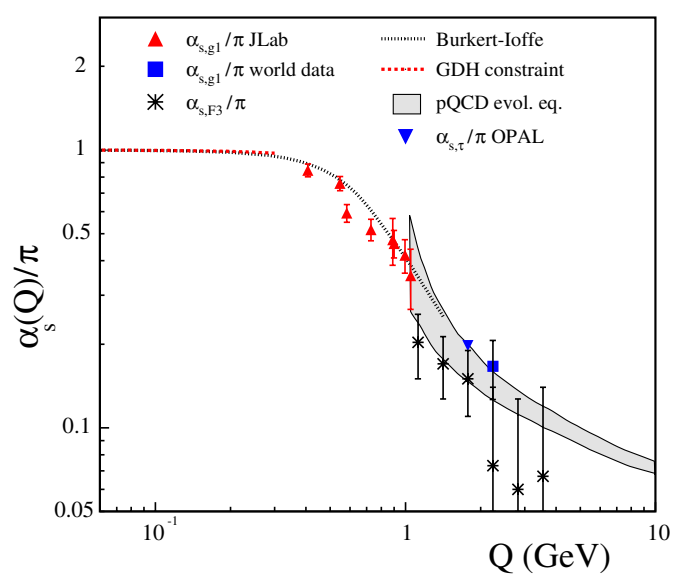

Fig. 18. Effective strong-coupling constant as a function of $Q^{2}$ derived from the $Q^{2}$-dependence of the Bjorken integral [23].

of very low momentum transfer with high precision to test the predictions of $\chi \mathrm{PT}$.

The first significant measurement of the $Q^{2}$-dependence of the Bjorken integral (see fig. 17) was made for $Q^{2}=(0.05-2.5)(\mathrm{GeV} / c)^{2}$. Remarkably, pQCD-based $Q^{2}$ evolution matches the data down to a $Q^{2}$ of about $0.7(\mathrm{GeV} / c)^{2}$. Deur et al. [23] have made an interesting interpretation of the $Q^{2}$-dependence of the Bjorken integral in terms of an effective strong-coupling constant $\alpha_{\text {eff }}\left(Q^{2}\right)$ (see fig. 18). Again, there is evidence for a transition occurring around $Q^{2}=1(\mathrm{GeV} / c)^{2}$.

\section{Explore the limits of our understanding of nuclear structure}

As described above, experiments at Jefferson Lab are providing essential new insights into nucleon structure. In a very similar way, the precision, spatial resolution, and interpretability of experiments performed using electromagnetic probes are being used to address long-standing issues in nuclear physics, including specifically nucleon-nucleon correlations and the identification of the limits of our understanding of finite nuclei.

\subsection{Correlations in nuclei}

Nucleon-nucleon correlations have been a subject of great interest since the beginnings of the field. In his fabled "bible" on nuclear physics, Hans Bethe estimated that these correlations should be of scale a third of what one observes in nuclear physics, and indeed they are. However, finding clear, interpretable evidence for these correlations has been a real challenge to experimentalists.

The previous generation of $\left(e, e^{\prime} p\right)$ experiments carried out at Saclay, NIKHEF, and Mainz explored the spectral function strength for low-lying shells. Only about $2 / 3$ of the strength anticipated from a simple shell model was found. However, the interpretability of these measurements was limited by the uncertainties introduced by the 


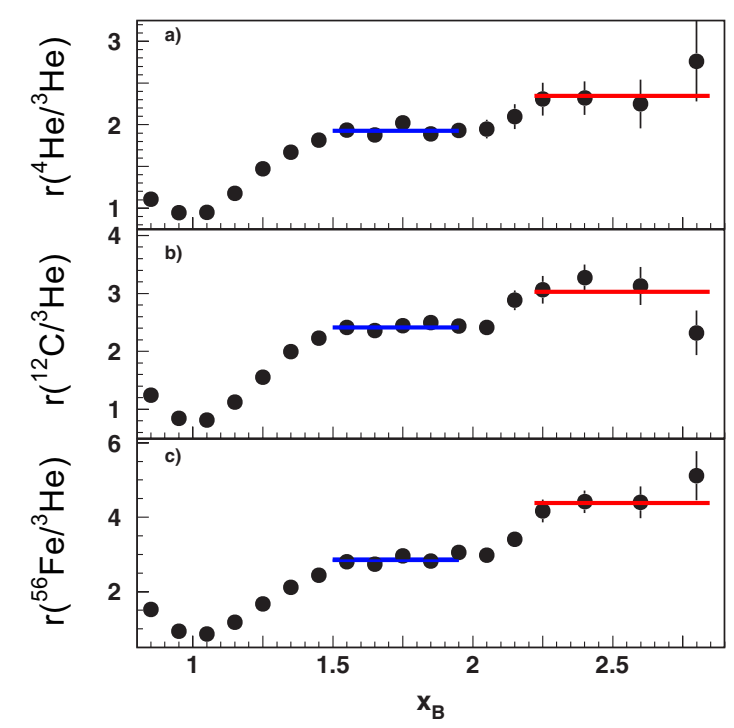

Fig. 19. Ratio of inelastic scattering cross sections off nuclei relative to ${ }^{3} \mathrm{He}$ as a function of Bjorken- $x$ [24].

corrections necessary for the final-state interactions of the knocked-out protons.

A new approach to nucleon-nucleon correlations avoids this problem by comparing the ratio of inelastic electron scattering off ${ }^{4} \mathrm{He},{ }^{12} \mathrm{C}$, and ${ }^{56} \mathrm{Fe}$ to ${ }^{3} \mathrm{He}$ in a kinematical regime where the scattering is basically from the quarks within the nucleons, and the scattering from the nucleons as coherent objects is highly suppressed. These data (see fig. 19) tell us that at any given moment the number of correlated nucleons in ${ }^{4} \mathrm{He},{ }^{12} \mathrm{C}$, and ${ }^{56} \mathrm{Fe}$ is $\sim 0.3, \sim 1.2$, and $\sim 6.7$, respectively. So about $10 \%$ of the time a nucleon is involved in a nucleon-nucleon correlation. The measurements further show that three-nucleon correlations are clearly present (at $x>2$ ), and about an order of magnitude smaller than two-nucleon correlations.

Another approach [25] to the study of correlations is to search explicitly for the strength that was identified as "missing" in the last generation of $\left(e, e^{\prime} p\right)$ experiments. We are using the $\left(e, e^{\prime} p\right)$ reaction at high momentum transfers and high missing energies, a region that was simply not accessible at the lower-energy, high duty-factor facilities previously available. The missing strength was, indeed, found (see fig. 20), and agrees rougly with the predictions of Correlated Basis Function theory (although the momentum distribution is not described correctly in detail).

In a third study correlated pairs have been measured directly in the ${ }^{3} \mathrm{He}\left(e, e^{\prime} p p\right) n$ reaction. In this experiment, the absorption of the virtual photon kicks out a proton, and the opening angle of the remaining pair shows a backto-back peak. One can infer from the data the shape of the pair momentum distribution.

Similar, though somewhat less direct, information can be obtained from examining the ${ }^{3} \mathrm{He}\left(e, e^{\prime} p\right) X$ reaction at very high missing momentum. Significant strength above what is predicted by PWIA has been observed (see fig. 21). The quantitative understanding of the results is work in progress.

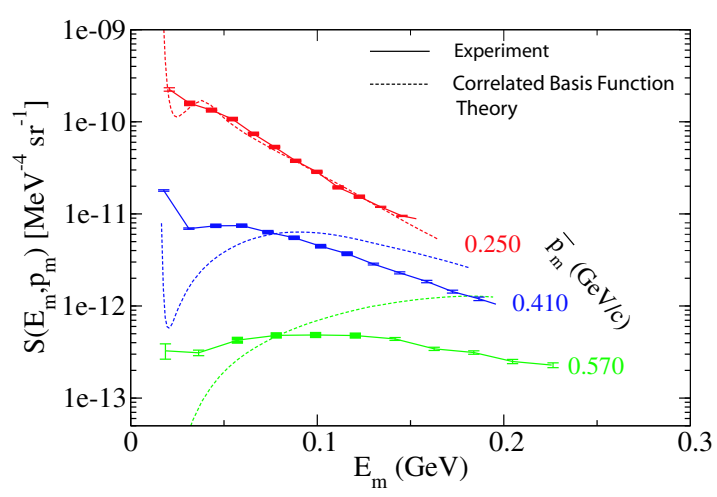

Fig. 20. Spectral function for $\left(e, e^{\prime} p\right)$ at high momentum transfer and high missing energy [25].

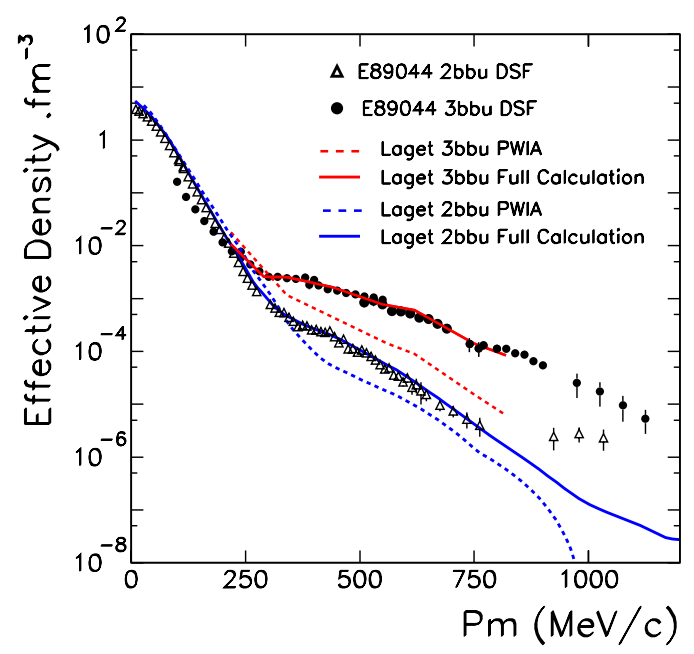

Fig. 21. Effective nucleon density for the ${ }^{3} \mathrm{He}\left(e, e^{\prime} p\right) X$ reaction as a function of missing momentum. 2bbu stands for two-body breakup [26].

\subsection{The limits of our understanding of finite nuclei}

One of the key issues that motivated the construction of CEBAF was our desire to gain insight into the question of where the description of nuclei based on nucleon and meson degrees of freedom fails and the underlying quark degrees-of-freedom must be taken into account. Data on the elastic scattering from the deuteron and highenergy photodisintegration, together with accurate theoretical calculations, are providing the answers.

We begin with the elastic scattering form factors for the deuteron. The theory is in an advanced state: we use the best $a b$ initio calculation of the structure of the deuteron with a potential $V_{N N}$ determined from a fit to $\mathrm{N}-\mathrm{N}$ phase shifts, and then add exchange currents and relativistic corrections. The data set for the deuteron elastic form factors demonstrate the technical accomplishments of modern accelerators and equipment: elastic e-D scattering has been measured down to cross sections characteristic of $\nu$-scattering!

The data for the electric and the magnetic form factors, and for the tensor polarization (see fig. 22) demonstrate that conventional nuclear theory works up to $Q^{2}$ of 

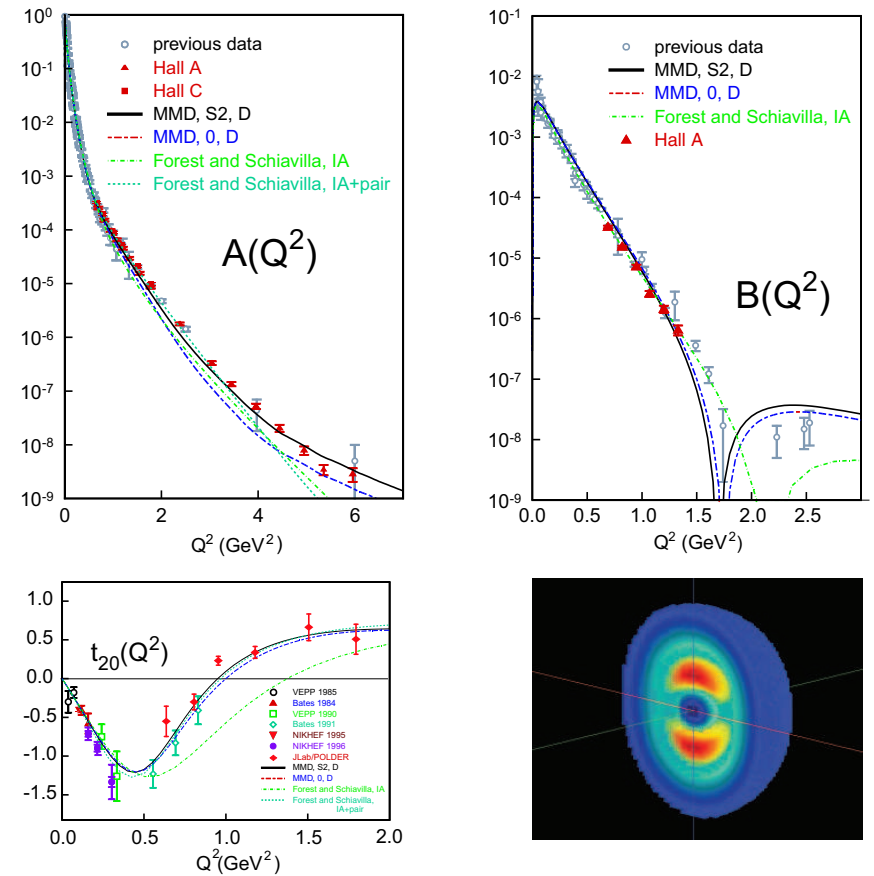

Fig. 22. Electric and magnetic form factors of the deuteron (top panels) and the deuteron tensor polarization (lower left) together with the intrinsic shape of the deuteron inferred from these data (lower right) (adapted from [27]).

about $2(\mathrm{GeV} / c)^{2}$, i.e. the nucleon-based picture is still valid at distance scales of about one half the size of a nucleon. Why, we do not know; none of us expected it to work that well before the experiments were undertaken. The shape of the deuteron derived from the form factor data is also shown in fig. 22; one can see clearly that the nucleon spins are aligned "end-to-end" (resulting in a "dumbell"-shaped distribution) rather than anti-parallel (which would have yielded a "donut" shape).

The photodisintegration of the deuteron was one of the first experiments done in nuclear physics (at energies of only a few $\mathrm{MeV}$ ) and also one of the most recent ones (now at energies approaching $6 \mathrm{GeV}$ ). The reaction probes internal nucleon momenta well beyond those accessible in electron scattering because of the momentum mismatch between the photon and the nucleon. In a parton-based description of the reaction, one expects the cross section to scale like $s^{-11}$, where $s$ is the CM energy squared. The data (see fig. 23) demonstrate that $s^{-11}$ scaling of the cross section is reached at photon energies which change with the proton center-of-mass angle. The transition occurs consistently at a transverse momentum of about $(1.0-1.3) \mathrm{GeV} / c$, which shows that below $\sim 0.2 \mathrm{fm}$ the nucleon-meson description of the deuteron is no longer valid, and a parton-based description is more appropriate. A more recent experiment [28] using CLAS has extended these data to include angular distributions for a broad range of energies; the data is described by a quark-gluon string model.

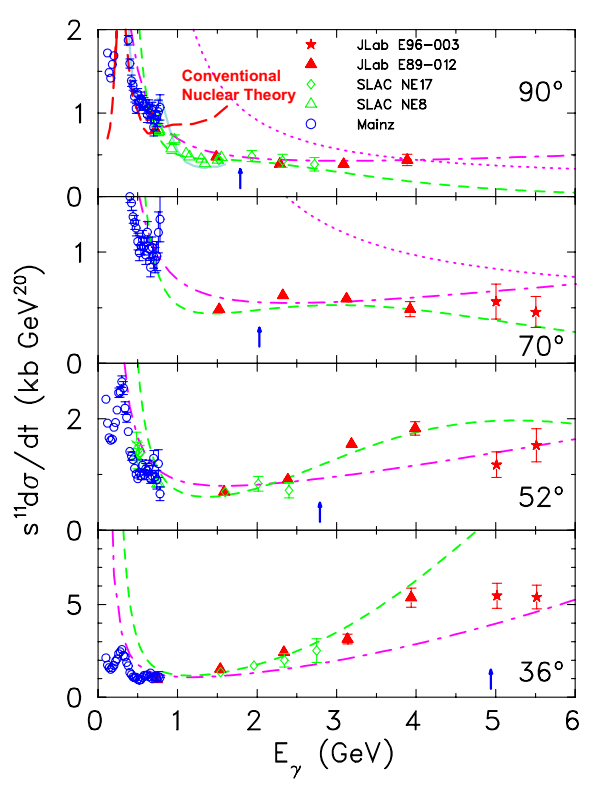

Fig. 23. Cross sections for deuteron photodisintegration. The energies associated with a transverse momentum of $1.37 \mathrm{GeV} / c$ are indicated with a blue arrow in each panel [29].

\section{Summary}

The CEBAF accelerator at JLab is fulfilling its scientific mission to understand how hadrons are constructed from the quarks and gluons of QCD, to understand the QCD basis for the nucleon-nucleon force, and to explore the transition from the nucleon-meson to a QCD description.

Its success is based on the firm foundation of experimental and theoretical techniques developed world-wide over the past few decades, on complementary data provided by essential lower-energy facilities, such as MAMI, and on the many insights provided by the scientists we are gathered here to honor.

It is a pleasure to acknowledge the assistance of Bernhard Mecking in the development of this article, and thoughtful comments on the manuscript from Volker Burkert, Kees de Jager, John Domingo, and Rolf Ent. This work has been supported through The Southeastern Universities Research Association, Inc., which operates the Thomas Jefferson National Accelerator Facility under Contract No. DE-AC05-84150 with the U.S. Department of Energy.

\section{References}

1. C. Hyde-Wright, C.W. de Jager, Annu. Rev. Nucl. Part. Sci. 54, 217 (2004) and references therein.

2. J. Friedrich, T. Walcher, Eur. Phys. J. A 17, 607 (2003).

3. J.J. Kelly, AIP Conf. Proc. 698, 393 (2004).

4. T.M. Ito et al., Phys. Rev. Lett. 92, 102003 (2004).

5. D.T. Spayde et al., Phys. Lett. B 583, 79 (2004).

6. F.E. Maas et al., Phys. Rev. Lett. 94, 152001 (2005).

7. F.E. Maas et al., Phys. Rev. Lett. 95, 022002 (2004).

8. F. Maas, these proceedings. 
9. K.A. Aniol et al., Phys. Rev. Lett. 96, 022003 (2006).

10. K.A. Aniol et al., Phys. Lett. B 635, 275 (2006).

11. K.A. Aniol et al., Phys. Lett. B 509, 211 (2001).

12. K.A. Aniol et al., Phys. Rev. C 69, 065501 (2004).

13. D.S. Armstrong et al., Phys. Rev. Lett. 95, 092001 (2005).

14. T. Horn, private communication for the $\mathrm{F}_{\pi}$ collaboration.

15. V.D. Burkert, Eur. Phys. J. A 17, 303 (2003).

16. L.C. Smith, Invited Talk, Japan-US Workshop on Electromagnetic Meson Production and Chiral Dynamics, Osaka, Japan (April 2005).

17. CLAS Collaboration (V.D. Burkert), Int. J. Mod. Phys A 20, 1531 (2005).

18. I. Aznauryan talk at $N^{*} 2005$, Tallahasse, $F L$ (October 2005), to be published.

19. X. Zheng et al., Phy. Rev. Lett. 92, 012004 (2004).
20. CLAS Collaboration (K.V. Dharmawardane et al.), submitted to Phys. Rev. Lett. (2006).

21. A. Thomas, these proceedings.

22. A. Deur, $13^{\text {th }}$ Int. workshop on Deep Inelastic Scattering (DIS2005), AIP Conf. Proc. 792, 969 (2005).

23. A. Deur, V. Burkert, J.P. Chen, W. Korsch, arXiv:hep$\mathrm{ph} / 0509113$.

24. K. Egiyan et al., Phys. Rev. Lett. 96, 082501 (2006).

25. D. Rohe et al., Phys. Rev. Lett. 93, 182501 (2004).

26. F. Benmokhtar et al., Phys. Rev. Lett. 94, 082305 (2005).

27. M. Garçon, J.W. Van Orden, Adv. Nucl. Phys. 26, 293 (2001) and references therein.

28. M. Mirazita et al., Phys. Rev. C 70, 014005 (2004).

29. E.L. Schulte et al., Phys. Rev. Lett. 87, 102302 (2001). 